\title{
Origin of the ankle in the ultra-high energy cosmic ray spectrum and the extragalactic protons below it
}

\author{
Glennys R. Farrar* \\ Center for Cosmology and Particle Physics, New York University, NY 10003, USA \\ E-mail: gf25@nyu. edu

\section{Michael Unger} \\ Center for Cosmology and Particle Physics, New York University, NY 10003, USA \\ Institut für Kernphysik, Karlsruher Institut für Technologie, 76021 Karlsruhe, Germany \\ E-mail: mu495@nyu.edu

\section{Luis A. Anchordoqui} \\ Department of Physics and Astronomy, Lehman College at CUNY, NY 10468, USA \\ Department of Physics, Graduate Center, City University of New York, NY 10016, USA \\ Department of Astrophysics, American Museum of Natural History, NY 10024, USA \\ E-mail: laa410@nyu. edu
}

\begin{abstract}
The sharp change in slope of the ultra-high energy cosmic ray (UHECR) spectrum around $10^{18.6} \mathrm{eV}$ (the ankle), combined with evidence of a light but extragalactic component near and below the ankle which evolves to intermediate composition above, has proved exceedingly challenging to understand theoretically. We show that for a range of source conditions, photodisintegration of ultra-high energy nuclei in the region surrounding a UHECR accelerator naturally accounts for the observed spectrum and composition of the entire extragalactic component, which dominates above about $10^{17.5} \mathrm{eV}$. The mechanism has a clear signature in the spectrum and flavors of neutrinos.
\end{abstract}

The 34th International Cosmic Ray Conference,

30 July- 6 August, 2015

The Hague, The Netherlands

\footnotetext{
* Speaker.
} 


\section{Introduction}

The cosmic ray spectrum spans roughly eleven decades of energy, $10^{9} \mathrm{eV} \lesssim E \lesssim 10^{20} \mathrm{eV}$. Continuously running monitoring using sophisticated equipment on high altitude balloons, satellites and ingenious installations on the Earth's surface explore the plummeting flux that decreases nearly three orders of magnitude per energy decade until eventually suffering a strong suppression around $10^{19.6} \mathrm{eV}[1,2]$. Close examination reveals several features in the spectrum, the three most important being: the steepening of the spectrum dubbed the "knee" occurring at approximately $10^{15.6} \mathrm{eV}$, from $J(E) \propto E^{-2.7 \pm 0.01}$ to $E^{-3.10 \pm 0.07}$ [3]; a less prominent "second knee", corresponding to a further softening $J(E) \propto E^{-3.52 \pm 0.19}$ around $10^{17.5} \mathrm{eV}$ [4]; a pronounced hardening of the spectrum at $E \approx 10^{18.6} \mathrm{eV}$, constituting the so-called "ankle" feature [1, 5].

The variations of the spectral index reflect various aspects of cosmic ray production, source distribution and propagation. The first and second knee have straightforward explanations, as reflecting the maximum energy of Galactic magnetic confinement or acceleration capability of the sources, both of which grow linearly in the charge $Z$ of the nucleus; the first knee being where protons drop out and the second knee where the highest- $Z$ Galactic CRs (GCRs) drop out. As the energy increases above the 2nd knee to the ankle, the composition evolves from heavy to light [6] while the CR arrival directions are isotropic to high accuracy throughout the range [7]. Finally, as the energy increases above the ankle, not only does the spectrum harden significantly, but the composition gradually becomes heavier (interpreting the data using conventional extrapolations of accelerator-constrained particle physics models) [8]. The Telescope Array Collaboration reports a composition consistent with pure proton, but their data are also consistent with the results of the Pierre Auger Observatory when systematic and statistical uncertainties are taken into account [9].

This observed evolution in the extragalactic CR (EGCR) composition and spectral index presents a major conundrum. A pure proton composition might be compatible with the observed spectrum of EGCRs [10] when allowance is made for experimental uncertainties in the energy scale and the fact that the real local source distribution is not homogeneous and continuous [11] (although the sharpness of the ankle is difficult to accommodate), but a pure proton composition is incompatible with the depth-of-shower-maximum $\left(X_{\max }\right)$ distributions observed by Auger [8] unless current extrapolations of particle physics are incorrect. On the other hand, models which fit the spectrum and composition at highest energies, predict a deep gap between the end of the GCRs and the onset of the EGCRs. Models can be devised to fill this gap, but fine-tuning is required to position this new population so as to just fit and fill the gap [12].

Here we offer a resolution to this conundrum, by showing that "post-processing" of UHECRs via photo-disintegration in the environment surrounding the source, can naturally explain the entire spectrum and composition. In our model, EGCRs below the ankle are predominantly protons knocked off higher energy nuclei in the region surrounding the accelerator, and the spectrum and composition above the ankle are predominantly dictated by the accelerator and propagation to Earth. The model makes distinctive predictions about the spectrum and flavor ratios of neutrinos, which should enable it to be tested. If the ankle and the protons below it arise on account of our mechanism, we obtain a new constraint on UHECR sources beyond the Hillas criterion and totalenergy-injection requirements, namely that the environment around the source has the conditions giving rise to the required amount of photo-disintegration. 


\section{Formation of the Ankle}

To illustrate the mechanism we have identified to create the ankle and generate extragalactic protons at lower energies, consider a system in which the accelerator is embedded in a photon field and the CRs are trapped by magnetic fields in this environment. It is standard to consider the impact of the photons that are encountered during the process of acceleration, in case those limit the maximum energy. The new feature here is to also allow for and explore the impact of interactions with photons in the surrounding medium. Examples could be the dusty torus surrounding an AGN or the ISM of the star-forming region surrounding most young pulsars. See also [13].

A simple analytic treatment is instructive. Details are derived in [14], but the essential simplifying assumptions are i) a CR either escapes without changing energy, with a rate $\tau_{\mathrm{esc}}$, or the CR interacts one or more times before escaping; ii) no energy is lost except through an interaction and whenever a nucleus interacts it loses one or more nucleons by photodisintegration or photopion production; in this case the nucleus loses a fraction of its energy corresponding to the reduction in its nuclear mass; iii) $\tau_{\mathrm{esc}}$ and $\tau_{\text {int }}$ are independent of position and depend only on $E, A, Z$ of the nucleus.

In this approximation the number of nuclei in a given energy bin with specified $A, Z$ decreases exponentially with time. The decay constant is $\tau=\left(1 / \tau_{\text {esc }}+1 / \tau_{\text {int }}\right)^{-1}$, where $\tau_{\text {esc }}$ and $\tau_{\text {int }}$ are the escape and interaction times respectively. A fraction $\eta_{\text {esc }}$ of the particles escape without interaction

$$
N_{\mathrm{esc}}=\int_{0}^{\infty} \frac{1}{\tau_{\mathrm{esc}}} N(t) d t=\frac{N_{0}}{1+\tau_{\mathrm{esc}} / \tau_{\mathrm{int}}} \equiv N_{0} \eta_{\mathrm{esc}}
$$

and the rest interact before escaping so $\eta_{\text {int }}=1-\eta_{\text {esc }}$. Note that $\eta_{\text {esc }}$ and $\eta_{\text {int }}$ depend only on the ratio of the escape and interaction times, but not on the absolute value of either of them.

The mechanism for generating an ankle-like feature can be explained most easily by considering the full dissociation of injected nuclei of mass $A^{\prime}$, to $A^{\prime}$ nucleons. If the escape and interaction times are both power laws in energy, $\tau_{\mathrm{esc}} \equiv a\left(E^{\prime} / E_{0}\right)^{\delta}$ and $\tau_{\text {int }} \equiv b\left(E^{\prime} / E_{0}\right)^{\beta}$, then

$$
\eta_{\mathrm{esc}}\left(E^{\prime}\right)=\left(1+R_{0}\left(E^{\prime} / E_{0}\right)^{\delta-\beta}\right)^{-1},
$$

where $R_{0}=a / b$ is the ratio of the escape and interaction time at reference energy $E_{0}$. Therefore, if $\delta>\beta$ the propagation in the source acts as a low-pass filter on the spectrum of injected particles leading to a cutoff in the spectrum at high energies. This situation typically occurs in stochastic shock acceleration scenarios where $\delta>0$, for non-thermal photon energy spectra with $\beta<0$. If, on the other hand, the escape times decrease with energy, as in the case of diffusion in turbulent magnetic fields, then it is possible to have $\delta<\beta$ leading to a high-pass filter for the energy spectrum of injected nuclei. In this case, the lower the energy the more nuclei interact, leading to an apparent hardening of the spectrum of escaping nuclei. The produced nucleons on the other hand have energies of $E^{*}=E^{\prime} / A^{\prime}$. These nucleons are most abundant at low energies and have a steeper spectrum $\propto\left(1-\eta_{\mathrm{esc}}\left(E^{*} A^{\prime}\right)\right)$. If interactions of these nucleons are negligible, the high-pass scenario leads naturally to an ankle-like feature separating the nucleonic fragments from the remaining nuclei, at the energy where the number of escaping nuclei is equal to the number of nucleons. 

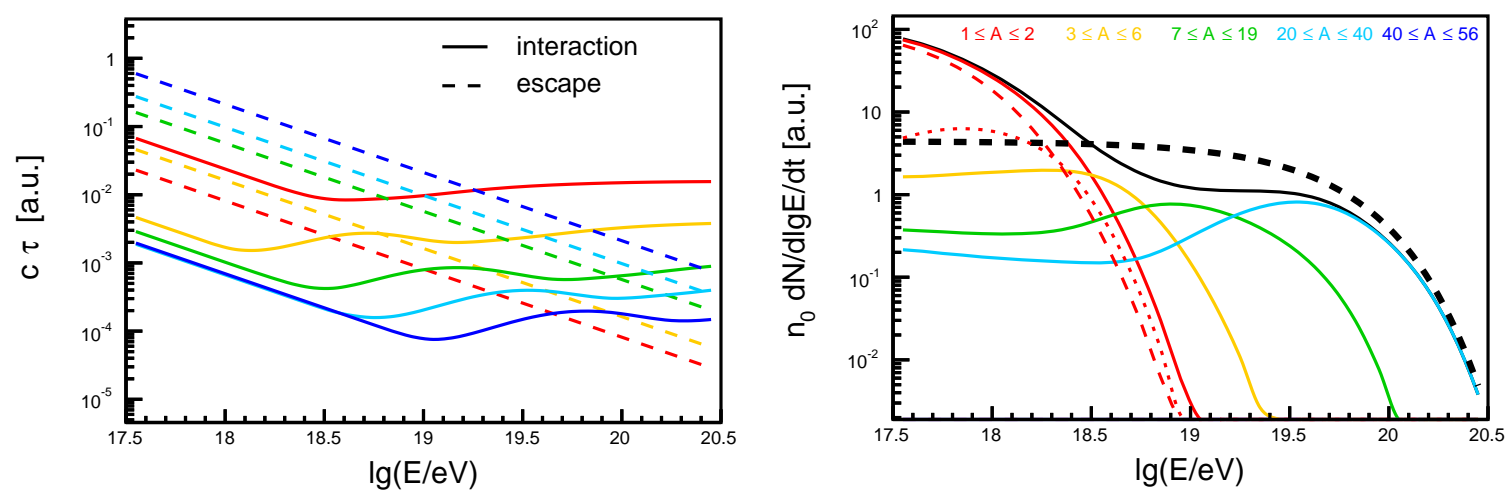

Figure 1: Left: Interaction and escape times for $A^{\prime}=1,4,14,26$ and 56 (bottom to top for escape and vice versa for interaction). Injected ${ }^{28} \mathrm{Si}$ flux (bold dashed) and escaping flux. Right: Mass groups are colorcoded with increasing mass from left to right. The thin black solid line denotes the sum of all escaping nuclei. Nucleons from photo-dissociation are shown as a thin dashed curve and nucleons from photo-pionproduction as dotted curve.

We consider two characteristic photon spectra. The first consists of broken power-law as a simplified representative of non-thermal emission. The second is a modified black-body spectrum. The interaction time for a nucleus with Lorentz factor propagating through the photon field with differential number density $n(\varepsilon)$ is given by [15]. Inspired by the energy dependence of the diffusion coefficient for the propagation in a turbulent magnetic field, we model $\tau_{\text {esc }}$ as a power law in rigidity $E / Z$

$$
\tau_{\mathrm{esc}}=\tau_{0}\left(\frac{E / Z}{E_{0}}\right)^{\delta} .
$$

The left panel of Fig 1 shows the escape and interaction times as a function of the CR energy (proton, $\mathrm{He}, \mathrm{N}, \mathrm{Si}$ and $\mathrm{Fe}$ ). We adopt $\delta=-1$, as for Bohm diffusion. We take a broken power-law photon spectrum, which peaks in the far infrared $\left(\varepsilon_{0}=50 \mathrm{meV}\right)$ and has slope parameters $\alpha=+\frac{5}{2}$ and $\beta=-2$. The normalization of the photon density and escape time, $n_{0}$ and $\tau_{0}$, are chosen so that the ratio of interaction and escape time at $10^{19} \mathrm{eV}$ for iron nuclei, $R_{19}^{\mathrm{Fe}}$, is 275 ; the interaction times are calculated including both photo-disintegration and photo-pion production.

The gross features of the energy dependence of the interaction times can be understood in the approximation of resonant interactions at center of mass energies of $\varepsilon_{\text {res }}^{\mathrm{CM}}$. At low cosmic-ray energies, reaching $\varepsilon_{\mathrm{res}}^{\mathrm{CM}}$ requires high photon energy $\left(\varepsilon>\varepsilon_{0}\right)$, so that the interaction time decreases with cosmic-ray energy as $\tau \propto E^{\beta+1}$. However, for high enough CR energy, the resonance can be reached in collisions with photons of $\varepsilon<\varepsilon_{0}$; from here, as the CR energy increases, the photon density decreases as $\varepsilon^{\alpha}$, and correspondingly the interaction times increase. The laboratory energy of the inflection point of the interaction times for a CR nucleus of mass $M$ is at $E=M \varepsilon_{\text {res }} /\left(2 \varepsilon_{0}\right)$. The inflection point of the photo-dissociation times can be seen as a dip in the right panel of Fig. 1, e.g. at around $10^{19.3} \mathrm{eV}$ for iron nuclei. At slightly higher energy photo-pion production becomes important, with the result that the energy dependence of the interaction time is roughly speaking an L-shaped curve in a log-log presentation. 

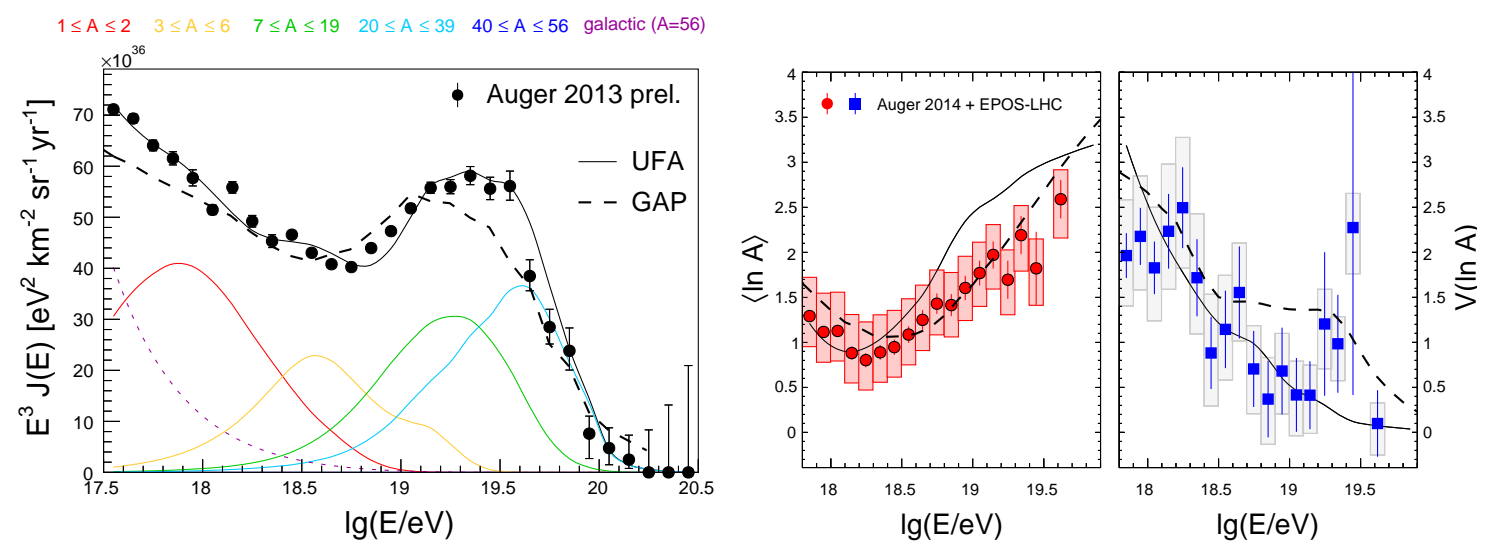

Figure 2: Spectrum and composition at Earth. Dots are data from the Pierre Auger Observatory [16, 8], error bars denote the statistical uncertainties and the shaded boxes in the center and right figure illustrate the experimental systematic uncertainties of the moments of $\ln A$. These composition estimates are based on an interpretation of air shower data with EPOS-LHC. The solid lines denote the predictions of our model and the dashed lines are the results from [24].

Using these energy-dependent interaction and escape times, we propagate nuclei through the source environment with the procedure described in [14].

As an example, we injected silicon nuclei, $A^{\prime}=28$, with a power law $E^{-1}$ and maximum energy $Z^{\prime} E_{\max }^{p}=3 \times 10^{19} \mathrm{eV}$. The unmodified spectrum and the spectrum of escaping nuclei are shown Fig. 1; the units are arbitrary, but the normalization in the two figures is the same. At low energies, the injected nuclei are depleted because $\tau_{\text {esc }} \gg \tau_{\text {int }}$, but the escaping nuclei follow the original spectral index, because in this example the interaction and escape times are parallel, as to be expected for $\delta=\beta+1$. Once the corner of the L-shape is reached, the fraction of escaping nuclei grows leading to an apparent hardening of the spectral index.

Even for the simple case of an injection of a single nuclear species into the source environment, we obtain a complex evolution of the mass composition with energy. At low energies the composition is dominated by knock-off nucleons whereas at high energies the composition becomes heavier as the ratio of escape to interaction time drops and more heavy nuclei can escape before interacting.

A qualitatively similar result is obtained when assuming a modified black-body spectrum, where the parameters $T=150 \mathrm{~K}$ and $\sigma=2$ have been chosen to match the peak and shape of the power-law spectrum used above. The corresponding interaction lengths and escaping spectra are shown in [14]. In both cases the spectrum of escaping particles displays an ankle-like feature from the transition between the soft spectrum of nucleons and the hard spectrum of nuclei. We have checked that the approximation of neglecting single-nucleon energy loss in the source environment is valid for the examples and parameter ranges discussed here. For a concrete astrophysical realization of this scenario, one must also check and if necessary include the effect of interactions with UHECRs with hadrons in the source environment. 


\section{Comparison to Data}

The model presented here is a very simple one, yet even so it offers a remarkably good accounting for the flux and composition at Earth as determined by the Pierre Auger Observatory. We compare to the measured flux from $10^{17.5} \mathrm{eV}$ to above $10^{20} \mathrm{eV}$ [16] and the mean and variance of the distribution of the logarithm of mass on top of the atmosphere, $\langle\ln A\rangle$ and $V(\ln A)[8,17]$.

We take as fiducial example, a hard UHECR injection spectrum $E^{-1}$ as expected for acceleration in young neutron stars [18] and use the broken power law photon spectrum with peak $\varepsilon_{0}$ in the far infrared at $\varepsilon_{0}=50 \mathrm{meV}$ and spectral indices $\alpha=+\frac{5}{2}$ and $\beta=-2$. We assume that the evolution of the sources follows the star formation rate [19] (see [20] for a model of cosmic rays above the ankle using a negative source evolution).

To keep the complexity of the model to a minimum here, we inject only a single nuclear species. The best description of the data is obtained with ${ }^{28} \mathrm{Si}$. Another free parameter is the maximum energy, for which the best fit gives a value of $Z^{\prime} \times 10^{18.5} \mathrm{eV}=3 \times 10^{19} \mathrm{eV}$.

The ratio of the escape time and the interaction time in the photo-disintegration region at a specified rigidity and composition is the only free parameter characterizing the photo-disintegration; the best fit corresponds to iron at $E=10^{19} \mathrm{eV}$ having an escape length 275 times its interaction length. The spectral index and normalization of the Galactic spectrum are free "nuisance" parameters as well. The best fit gives a spectral index of -4.2 and a contribution of about half of the flux at the lowest energy of the fit $\left(10^{17.5} \mathrm{eV}\right)$, fully compatible with indications from KASCADEGrande [21].

The resulting fit is shown in comparison to data in Fig. 2. There is a good overall agreement between the model and the data. The shape of the spectrum is described well, including the ankle and the flux suppression. The model also qualitatively reproduces the increase of the average logarithmic mass with energy and the decrease of its variance. Normalizing this model to the observed flux at Earth, we infer a comoving energy injection rate in CRs at $z=0$ and above $10^{17.5} \mathrm{eV}$ of $\dot{\varepsilon}_{17.5}=8.2 \times 10^{44} \frac{\mathrm{erg}}{\mathrm{Mpc}^{3} \mathrm{yr}}$.

To explore the sensitivity of the quality of the fit to uncertainties in the model and data, we have varied the parameters of the model and the general features are consistently reproduced (see [14] for details). Changing the extragalactic background light model from [22] which represents an upper limit, to the lower limit of [23], results in a similar description of the data with $A^{\prime}=23$ and an $R_{19}^{\mathrm{Fe}}$ increased by factor of 1.5 . Fixing $A^{\prime}=28$ and letting the spectral index float freely leads to a spectrum following $E^{-1.04}$. Changing the photon field from a broken power law to a modified black body prefers the mass to be at $A^{\prime}=27$ and reduces $R_{19}^{\mathrm{Fe}}$ by 0.7 . Concerning the photon field, we checked that we get acceptable fits for a peak photon energy in the range of 25 to $100 \mathrm{meV}$, values of $\beta$ in range of -2.5 to -1.5 , and that there is little sensitivity on $\alpha$. For the escape lengths, the power law index $\delta$ can be as large as -0.7 . Even slower escapes could probably be accommodated by a variation of $\beta$ at the same time.

We find that the best fit is obtained within the experimental systematics when shifting the energy scale up by $+1 \sigma_{\text {sys. }}=15 \%$ and by shifting $\langle\ln A\rangle$ and $V(\ln A)$ corresponding to a shift of the shower maximum by $-1 \sigma_{\text {sys. }} \approx-10 \mathrm{~g} / \mathrm{cm}^{2}$.

Using alternative models for the hadronic interactions of air showers (SIB YLL2.1 or QGSJETII04 instead of Epos-LHC), decreases the value of the $\langle\ln A\rangle$ data points by about $\langle\ln A\rangle=-0.6$ and 
leads to a worse fit of the data, within this limited exploration of parameter space. It is worthwhile noting that if the difference between models gives a fair estimate of the uncertainties of the mass determination in both directions, $\sigma_{\text {theo }}(\langle\ln A\rangle)= \pm 0.6$, then a hadronic interaction model that would give a larger mass estimate than EPOS-LHC would make this fiducial model fit even better.

A related analysis was presented in [24]. They explore a specific model of GRB internal shock acceleration and find that for sufficiently strong source evolution and properly chosen GRB model parameters, photo-disintegration on photons created within the GRB, can produce an ankle-like feature. The predictions of this model are shown as dahsed lines in Fig. 2.

The neutrino signals of our model are discussed in [14]. We emphasize the distinctive $\bar{v}_{e}$ enrichment due to beta decay of spallated neutrons.

\section{Conclusions}

We have exposed general conditions which give rise to an ankle-like feature in the source emission spectrum, with light composition below and increasingly heavy composition above, as called for by the data with conventional particle physics modeling. We illustrated the high quality of the fit which can be obtained to the data, with a fiducial model in which nuclei accelerated up to a maximum rigidity, with spectrum $\propto E^{-1}$, are subject to photo-disintegration in the vicinity of the accelerator before escaping for their journey to Earth. Such a scenario can be reasonably achieved in astrophysical sources, as will be discussed in a future publication. Very generally, when nuclei remain trapped in the turbulent magnetic field of the source environment their escape time can decrease faster with increasing energy than does their interaction time. When these general conditions are realized, only the highest energy particles can escape the source environment unscathed. In other words, the source environment acts as a high-pass filter on the spectrum of cosmic rays. Nuclei below the crossover energy scatter off the far-infrared photons in the source environment, with ejection of nucleons or alpha particles. This produces a steep spectrum of secondary nucleons. The superposition of the steeply falling nucleon spectrum with the harder spectrum of the surviving nuclear fragments creates an ankle-like feature in the total source emission spectrum. This occurs at an energy of about $10^{18.6} \mathrm{eV}$, for a large range of parameter values, reflecting the kinematics of the giant dipole resonance. The spectrum above the ankle exhibits a progressive transition to heavy nuclei, as the escape of non-interacting nuclei becomes efficient. Abundant production of $\bar{v}_{e}$ 's is a signature of this mechanism.

\section{Acknowledgments}

We would like to acknowledge many useful discussions with our colleagues of the Pierre Auger Collaboration. Furthermore we thank David Walz for his support regarding questions about CRPROPA. MU acknowledges the financial support from the EU-funded Marie Curie Outgoing Fellowship, Grant PIOF-GA-2013-624803. The research of GRF is supported in part by the U.S. National Science Foundation (NSF), Grant PHY-1212538 and the James Simons Foundation; she thanks KIPAC/SLAC for their hospitality. The research of LAA is supported by NSF (Grant CAREER PHY-1053663) and NASA (Grant NNX13AH52G); he thanks the Center for Cosmology and Particle Physics at New York University for its hospitality. 


\section{References}

[1] R. Abbasi et al. [HiRes Collaboration], Phys. Rev. Lett. 100, 101101 (2008).

[2] J. Abraham et al. [Pierre Auger Collaboration], Phys. Rev. Lett. 101, 061101 (2008).

[3] T. Antoni et al. [KASCADE Collaboration], Astropart. Phys. 24, 1 (2005).

[4] T. Abu-Zayyad et al., Astrophys. J. 557, 686 (2001).

[5] J. Abraham et al. [Pierre Auger Collaboration], Phys. Lett. B 685, 239 (2010).

[6] K. H. Kampert and M. Unger, Astropart. Phys. 35, 660 (2012).

[7] P. Abreu et al. [Pierre Auger Collaboration], Astropart. Phys. 34, 627 (2011); Astrophys. J. Suppl. 203, 34 (2012) Astrophys. J. 802, 111 (2015).

[8] A. Aab et al. [Pierre Auger Collaboration], Phys. Rev. D 90, 122005 (2014); Phys. Rev. D 90, 122006 (2014).

[9] R. Abbasi et al. [Pierre Auger and Telescope Array Collaborations], arXiv:1503.07540.

[10] V. Berezinsky, A. Z. Gazizov and S. I. Grigorieva, Phys. Rev. D 74, 043005 (2006).

[11] M. Ahlers, L. A. Anchordoqui and A. M. Taylor, Phys. Rev. D 87, 023004 (2013).

[12] T. K. Gaisser, T. Stanev and S. Tilav, Front. Phys. China 8, 748 (2013); R. Aloisio, V. Berezinsky and P. Blasi, JCAP 1410, 020 (2014); G. Giacinti, M. Kachelrieß and D. V. Semikoz, Phys. Rev. D 91, 083009 (2015).

[13] D. Allard and R. J. Protheroe, Astron. Astrophys. 502, 803 (2009); K. Kotera, D. Allard, K. Murase, J. Aoi, Y. Dubois, T. Pierog and S. Nagataki, Astrophys. J. 707, 370 (2009); A. Pe'er, K. Murase and P. Meszaros, Phys. Rev. D 80, 123018 (2009); K. Fang, K. Kotera and A. V. Olinto, Astrophys. J. 750, 118 (2012); K. Fang, K. Kotera and A. V. Olinto, JCAP 1303, 010 (2013); N. Globus, D. Allard, R. Mochkovitch and E. Parizot, Mon. Not. Roy. Astron. Soc. 4515270 (2015).

[14] M. Unger, G. R. Farrar and L. A. Anchordoqui, arXiv:1505.02153.

[15] F. W. Stecker, Phys. Rev. 180, 1264 (1969).

[16] A. Schulz for the Pierre Auger Collaboration, Proc. 33rd ICRC (2013), arXiv:1307.5059.

[17] P. Abreu et al. [Pierre Auger Collaboration], JCAP 1302, 026 (2013). E. J. Ahn for the Pierre Auger Collaboration, Proc. 33rd ICRC (2013), arXiv:1307.5059.

[18] P. Blasi, R. I. Epstein and A. V. Olinto, Astrophys. J. 533, L123 (2000) [astro-ph/9912240].

[19] B. E. Robertson, R. S. Ellis, S. R. Furlanetto and J. S. Dunlop, arXiv:1502.02024.

[20] A. M. Taylor, M. Ahlers and D. Hooper, arXiv:1505.06090 [astro-ph.HE].

[21] W. D. Apel et al. [KASCADE-Grande Collaboration], Phys. Rev. D 87, 081101 (2013).

[22] T. M. Kneiske, T. Bretz, K. Mannheim and D. H. Hartmann, Astron. Astrophys. 413, 807 (2004).

[23] T. M. Kneiske and H. Dole, Astron. Astrophys. 515, A19 (2010).

[24] N. Globus, D. Allard and E. Parizot, arXiv:1505.01377. 